\title{
EFECTO DE Ulva Spp. SOBRE EL CRECIMIENTO Y SUPERVIVENCIA DE Argopecten purpuratus EN LA BAHÍA DE PARACAS
}

\section{EFFECT OF Ulva spp. ON GROWTH AND SURVIVAL OF Argopecten purpuratus IN PARACAS BAY}

\author{
Daniel Arce Castro ${ }^{1}$ y Jaime Mendo ${ }^{2}$
}

\begin{abstract}
Resumen
Este estudio compara el crecimiento y supervivencia de Argopecten purpuratus (Lamarck, 1819) "concha de abanico" sembradas con diferentes densidades de Ulva spp., Linnaeus, 1753 en la bahía Paracas (Ica-Perú) durante el 7 agosto al 16 de octubre del 2011. Para ello se instalaron tres corrales cada uno con tres unidades experimentales de $1 \mathrm{~m}^{2}$ y en cada unidad se sembraron conchas de abanico de 40-50mm de altura a una densidad de 60 individuos $/ \mathrm{m}^{2}$. En dos de los corrales se sembró Ulva spp., con 10 (T2) y 20 (T3) kg/m² y el tercer corral fue considerado como testigo (T1), es decir, sin Ulva spp. Para evaluar el crecimiento, cada dos semanas se extrajeron al azar 10 individuos por cada repetición (30 por tratamiento) y se registró la altura y el peso seco del soma y la gónada. La supervivencia se evaluó contando los individuos muertos en periodos variables. Adicionalmente cada 30 minutos durante todo el experimento se registró la temperatura y oxígeno del fondo y diariamente el $\mathrm{pH}$, conductividad y corrientes se registraron en una zona media a los tratamientos. La clorofila $a$ se registró diariamente, para cada tratamiento.

Los resultados indican que la supervivencia, el crecimiento en altura y peso se vieron afectados por la presencia de Ulva spp. La concentración de oxígeno y clorofila $a$, mostraron mayores valores en los tratamiento con presencia de Ulva spp. Se concluyó que densidades mayores a $20 \mathrm{~kg}$ de Ulva spp. $/ \mathrm{m}^{2}$, afectan el crecimiento y supervivencia de Argopecten purpuratus, por lo que se recomienda su extracción.
\end{abstract}

Palabras clave: Argopecten purpuratus, Ulva spp., crecimiento, supervivencia, bahía Paracas, Perú.

\begin{abstract}
This study compares the growth and survival of juvenile Argopecten purpuratus (Lamarck, 1819) seeded with different densities of Ulva spp., in the bay of Paracas (Ica-Peru) between August 7 and October 16, 2011. For this purpose, three pens were installed each with three experimental units of $1 \mathrm{~m}^{2}$ and each experimental unit had a density of 60 scallops $/ \mathrm{m}^{2}$, with an average height of 40-50mm. In two of the pens Ulva spp., was introduced with 10 (T2) and 20 (T3) $\mathrm{kg} / \mathrm{m}^{2}$ and the third was considered witness (T1), ie without Ulva spp. To assess growth, every two weeks 10 scallops for each repetition (30 in each treatment) were randomly extracted their individual height was recorded and dry weight and gonad somatic tissue were sampled. Survival was assessed by counting the dead individuals in varying periods. During the entire experiment temperature and bottom oxygen were assessed every 30 minutes while $\mathrm{pH}$, conductivity and currents were daily assessed at noon in the treatments middle zone. Chlorophyll a was measured daily, for each treatment. The results indicate that survival, height and weight were affected by the presence of Ulva spp. Oxygen concentration and chlorophyll $a$, showed higher concentration in the presence of Ulva spp. It was concluded that higher than $20 \mathrm{~kg} \mathrm{spp} / \mathrm{m} 2$ densities of Ulva affect growth and survival of A. purpuratus, so a removal is recommended.
\end{abstract}

Key words: Argopecten purpuratus, Ulva spp., growth, survival, Paracas Bay, Perú.

\section{Introducción.}

La playa Atenas, en la bahía Paracas, Ica-Perú, es una de las zonas concesionadas para el desarrollo del cultivo de concha de abanico (A. purpuratus) en el Perú. Por ser una bahía somera los maricultores han optado por el engorde en sistemas de fondo que básicamente, consiste en cercar un área con malla, usando como lastres mangas rellenas con piedras y como flotadores boyas o botellas de plástico.

Uno de los problemas que presenta el engorde de esta especie en esta bahía, es la permanente colonización de macroalgas en especial las del género 
Ulva spp., las cuales pueden vivir fijas o flotando libremente (Hayden \& Waaland, 2004), a las que los maricultores de la zona atribuyen la causa de bajas tasas de crecimiento y supervivencia.

Estas especies han sido descritas como oportunistas, e invasoras a nivel mundial (Valiela et al., 1997; Jousson et al., 2000.; Meinesz et al., 2001) debido a su morfología del talo (delgado e indiferenciado), las tasas de crecimiento rápido y rápida aceptación de los nutrientes inorgánicos. Se caracterizan por absorber de manera eficiente el nitrógeno, incluso a bajas concentraciones (Hein et al., 1995.; Pedersen \& Borum, 1997.; Naldi, 2002). Así mismo, tienen la capacidad de almacenar reservas de nitrógeno en las células aprovechando episodios periódicos de alta disponibilidad de nitrógeno, que se producen en muchos ambientes costeros, como mencionan Fong et al. (1994), y Peckol et al. (1994), llegando a ser importantes productores primarios en ensenadas costeras o las causantes de desalojar otras especies.

Frente a la invasión masiva de Ulva spp., acompañada del supuesto de que las macroalgas generan pérdidas en la productividad del cultivo, los maricultores las extraen masivamente y las arrojan en otras zonas dentro de la bahía lo cual les genera un costo extra en la producción de $A$. purpuratus.

Al respecto, hasta la fecha no existen trabajos en el Perú que expliquen esta interacción específica de Ulva spp., y A. purpuratus. Por esta razón se planteó este estudio que tiene por objetivo determinar el efecto que produce la abundancia de Ulva spp., sobre el crecimiento y supervivencia de la concha de abanico.

\section{Materiales y métodos.}

El presente estudio se desarrolló en la concesión de cultivo de la empresa Inversiones Prisco S.A.C (13 49' 12.2' ' LS; 76 ${ }^{\circ} 18^{\prime}$ 02.8' LW) (Figura1), durante un periodo de 10 semanas, del 8 de agosto al 16 de octubre del 2011.

Diseño experimental.

Diseño e instalación del experimento.

Se utilizó un diseño completamente al azar (DCA

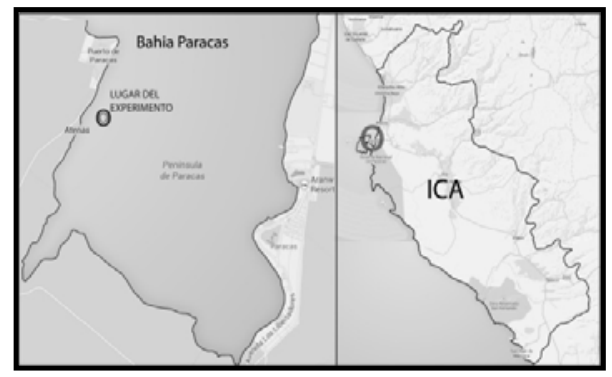

Figura 1. Mapa de ubicación del lugar donde se desarrolló el experimento en la playa Atenas, bahía Parcas, Ica-Perú. clásico o paramétrico), teniendo como factor la densidad de Ulva spp., bajo 3 niveles o tratamientos: $\mathrm{T} 1=\sin$ Ulva spp., o testigo, $\mathrm{T} 2=10 \mathrm{~kg}$ de Ulva spp. $/ \mathrm{m}^{2}$ y $\mathrm{T} 3=20 \mathrm{~kg}$ de Ulva spp. $/ \mathrm{m}^{2}$.

Para ello se construyeron tres corrales de $4 \mathrm{~m}^{2}$ de área y de malla anchovetera y como lastre en la parte inferior una manga de malla rellenas de piedras. Cada corral fue dividido en 4 subunidades de $1 \mathrm{~m}^{2}$. Los corrales se instalaron a una profundidad de $5 \mathrm{~m}$, a $200 \mathrm{~m}$ de la orilla sobre un sustrato mixto de conchuelas, arena y fango y a una distancia de $5 \mathrm{~m}$ entre ellos, con la finalidad que estos se encuentren bajo las mismas condiciones bio-oceanograficas.

En cada corral 3 unidades fueron usadas para las repeticiones y la cuarta unidad sirvió para la reposición de individuos sacrificados por los muestreos y/o pérdida por mortalidad, a fin de mantener la densidad de $A$. purpuratus constante en los tratamientos.

Una vez instalados los corrales se recolectaron frondas vivas de Ulva spp., que se encontraron libres en la misma concesión, las cuales fueron introducidas en cada corral o tratamiento a una biomasa de 0 (T1, testigo), 10 (T2) y $20 \mathrm{Kg} / \mathrm{m}^{2}$ (T3). Posteriormente se recolectaron un total de 810 individuos de $A$. purpuratus de la misma concesión entre $40 \mathrm{~mm}$ y 52 $\mathrm{mm}$ de altura, con una media de $46 \mathrm{~mm}$, de las cuales 90 conchas fueron seleccionadas al azar para realizar el muestreo inicial de peso seco. Las 720 conchas restantes se introdujeron de forma aleatoria 240 individuos en cada tratamiento, es decir $60 \mathrm{ind} / \mathrm{m}^{2}$ en cada unidad experimental. (Figura 2).

Crecimiento y supervivencia de juveniles.

El crecimiento se evaluó cada dos semanas, a través de la medición de la talla (altura) y peso seco del soma y gónada (g). Para ello en cada muestreo se sacrificaron 10 individuos al azar por repetición, 30 por tratamiento (90 en total), los cuales fueron

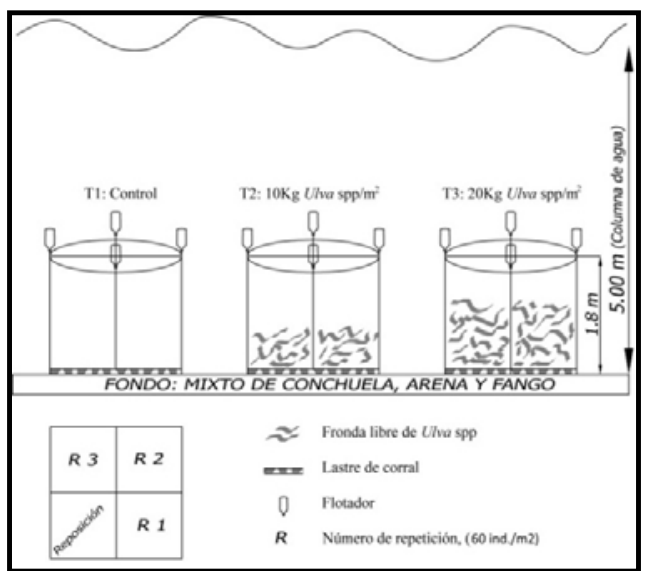

Figura 2. Esquema de la instalación del experimento en la playa Atenas, bahía de Paracas. 
reemplazados con individuos de la unidad de reposición y marcados con un corte en la valva, para evitar su selección para muestreos posteriores. Los individuos extraídos de la unidad de reposición eran a su vez reemplazados con individuos de la misma población y de tamaños similares.

Las muestras fueron llevadas en una bolsa térmica al Laboratorio de Recursos y Medio Ambiente de la Facultad de Pesquería de la Universidad Nacional Agraria la Molina, donde se registró la altura de las valvas con un malacómetro. El soma y gónada se colocaron en una estufa a $90^{\circ} \mathrm{C}$ por $25 \mathrm{~h}$ y se determinó el peso seco con ayuda de una balanza analítica de 0.0001g de precisión marca Acculab Atilon serie Analytical lab balances. Cabe mencionar que la parte somática corresponde al músculo abductor más las vísceras (manto + branquias + parte blanda).

La supervivencia de $A$. purpuratus se evaluó en intervalos variables debido a la disponibilidad de tiempo del personal de apoyo y se determinó mediante la extracción y conteo de todos los individuos (incluyendo los de reposición) de cada unidad experimental. Así mismo se realizó la reposición inmediata para cada repetición y cada tratamiento, los cuales fueron previamente marcados, haciendo unos pequeños cortes a las valvas y así evitar que fueran seleccionadas en los siguientes muestreos y mantener la densidad de $A$. purpuratus a lo largo de todo el experimento.

Medición de parámetros ambientales.

Las variables abióticas como temperatura $y$ oxígeno se registraron con ayuda de un datalogger de la marca RBR, de $0.001{ }^{\circ} \mathrm{C}$ y $0.001 \mathrm{ml} / \mathrm{L}$ de precisión los cuales fueron colocados a $30 \mathrm{~cm}$ del fondo en los Tratamiento T1 y T2.

La velocidad de la corriente $(\mathrm{cm} / \mathrm{s})$, se registró con un correntómetro, de marca General Oceanics INC, la conductividad eléctrica (CE) y el $\mathrm{pH}$ se registraron con ayuda de un multiparámetro digital de la marca HACH modelo HQ40D, siendo registrados al mediodía en un punto medio a los tratamientos.

La salinidad se determinó utilizando la relación encontrada por Bodelón, et al. (1994), entre la conductividad eléctrica $(\mathrm{mS} / \mathrm{cm})$ y la salinidad $(\mathrm{g} / \mathrm{L})$ :

$$
\log _{10}(\mathrm{Sal})=-0.175+1.0053 \log _{10}(\text { Cond } \mathrm{E})
$$

Con respecto a los parámetros bióticos, se realizaron medidas de concentración de clorofila $a$ $(\mu \mathrm{g} / \mathrm{L})$ con ayuda de un fluorómetro de la marca Turner Designs, modelo C-3, el cual durante las primeras cuatro semanas fue introducido al mediodía en cada tratamiento por un periodo de 5 minutos. Las siguientes semanas el registro fue continuo, con el fin de conocer la variación horaria.
Procedimientos de análisis de Datos.

Luego de obtener los datos de los muestreos se procedió a realizar diferentes pruebas estadísticas de acuerdo Cappelletti (1992) y así poder cuantificar el efecto de la densidad de Ulva spp., en el crecimiento y/o supervivencia.

Al cumplir la variable de estudio el supuesto de normalidad de Anderson-Darling y el de homogeneidad de variancias de Bartlett, se realizó un Análisis de variancia (ANVA) y se determinó el Coeficiente de Variación, para poder determinar el grado de homogeneidad de los resultados. Cuando el ANVA mostraba diferencia entre tratamientos, se aplicó la prueba de Tukey para comparar dicha variable.

\section{Resultados.}

Parámetros ambientales.

El T3 superó en $0.13^{\circ} \mathrm{C}$ a la media del $\mathrm{T} 1$, la temperatura mostró una tendencia decreciente con un incremento de variación día-noche, así mismo tendió a incrementarse durante el día llegando a su máximo a las 6.00 PM horas, para luego disminuir, llegando a su mínima a las 6:00 horas, (Figura 3-A y Anexo 1).
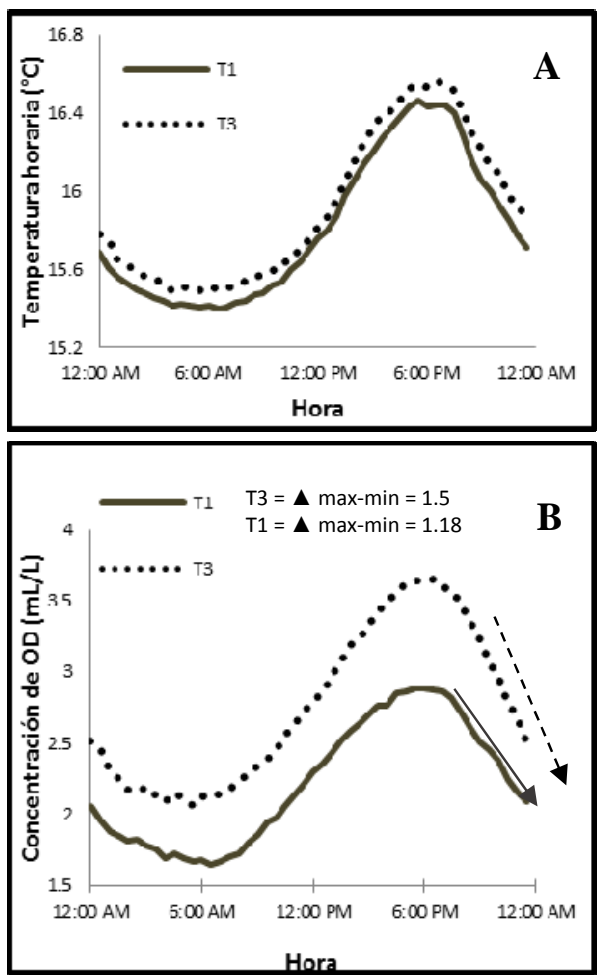

Figura 3. Temperatura horaria media $\left({ }^{\circ} \mathrm{C}\right)$ en T1 y T3 (A) y Concentración de oxígeno horaria (mL/L), en T1 y T3 (B), playa Atenas, bahía de Paracas, 8 de agosto al 16 de octubre 2011. 
Se presentó mayor concentración y variación de oxígeno disuelto en el T3, siendo más evidente en las últimas etapas $(\mathrm{T} 1=1.04 \mathrm{~mL} / \mathrm{L}, \mathrm{ds}=0.48$ y T3 $=2.56$

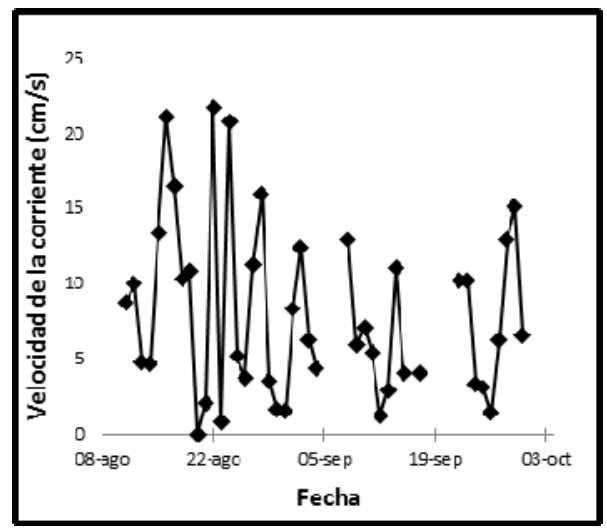

Figura 4. Velocidad de la corriente diaria cerca del fondo, en un intervalo de 5 minutos al mediodía, playa Atenas, bahía de Paracas.
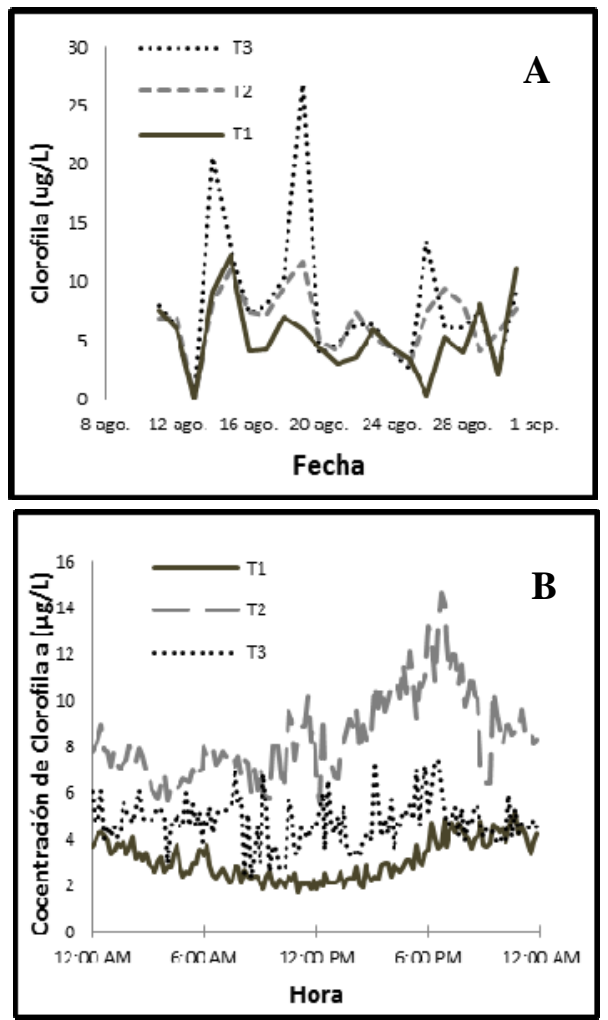

Figura 5. Concentración de clorofila $a$ $(\mu \mathrm{g} / \mathrm{L})$ diario, registrados al mediodía, (A) y concentración de Clorofila a promedio horaria $(\mu \mathrm{g} / \mathrm{L})(\mathrm{B})$, playa Atenas, bahía de Paracas, 10 al 23 de setiembre y de 2 al 16 de octubre del 2011.
$\mathrm{mL} / \mathrm{L}, \mathrm{ds}=1.10)$. La concentración de oxígeno tendió a incrementarse durante el día llegando a su máxima a las 6:00 PM horas y mínimas a las 6:00 horas (Figura 3-B).

Los valores de la velocidad de corrientes al mediodía fueron variables, registrando el mes de agosto, el mínimo $(0 \mathrm{~cm} / \mathrm{s}$, día 20$)$ y máximo $(21.75$ $\mathrm{cm} / \mathrm{s}$ día 22), (Figura 4).

Del registro de la concentración de clorofila $a$ al mediodía encontramos una mayor concentración y variación en los T2 y T3 (Figura 5A). De igual forma para los registros horarios se encontró una mayor variación en los tratamientos con presencia de Ulva spp. Así mismo la clorofila $a$ varia ligeramente a lo largo del día, llegando a sus mínimos valores al mediodía y sus máximos a la media noche, no siendo este comportamiento tan evidente en el T2 y T3 (Figura 5B).

El pH y la conductividad eléctrica no sufrieron grandes variaciones, el valor de $\mathrm{pH}$ varió de 7.4 a 8 y la conductividad osciló entre 52.9 y $53.8 \mathrm{mS} / \mathrm{cm}$. Lo que es equivalente a 36 y $36.7 \mathrm{gr} / \mathrm{L}$ de salinidad.

La Figura 6A muestra la altura valvar media, donde el $\mathrm{T} 1=61.5, \mathrm{~T} 2=59$ y $\mathrm{T} 3=54.5 \mathrm{~mm}$. En

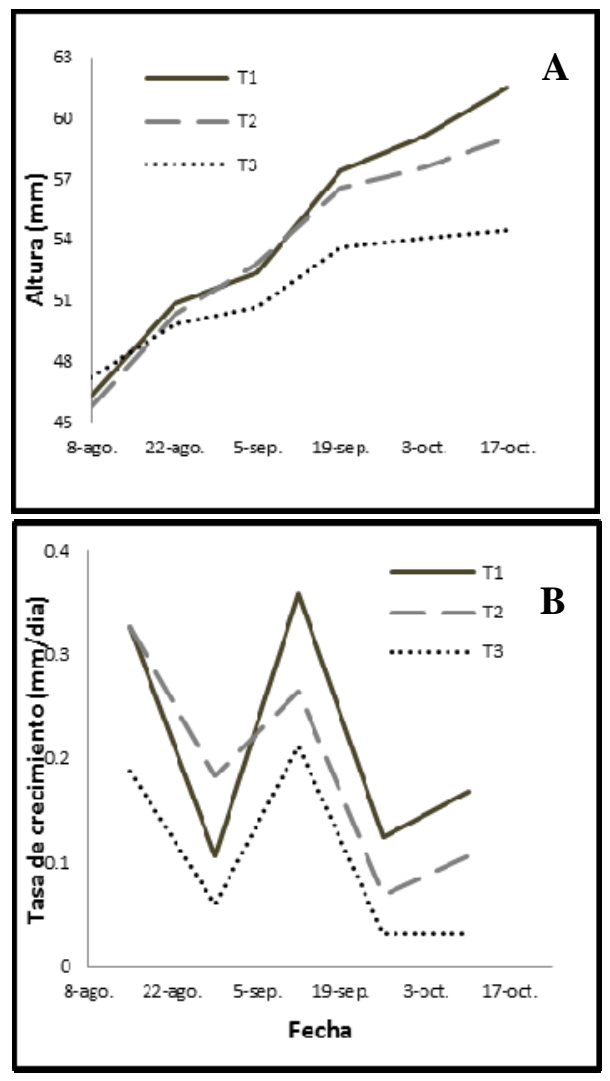

Figura 6. Altura valvar media (mm) (A) y Tasa de crecimiento de la altura ( $\mathrm{mm} /$ día) (B) de A. purpuratus, playa Atenas, bahía de Paracas. 
contraste, en la Figura $6 \mathrm{~B}$ se observa la tasa de crecimiento valvar, donde el T1 fue el que presentó la mayor tasa $(\mathrm{T} 1=0.22 ; \mathrm{T} 2=0.19 ; \mathrm{T} 3=0.11 \mathrm{~mm} /$ día $)$. Siendo el T1 significativamente diferente del T2 a partir de la tercera quincena y del T3 desde la segunda quincena. (Ver Anexo 2, Tabla 1).

La Figura 7A, muestra el crecimiento en peso seco del soma, donde el T1 mostro un crecimiento similar al T2, superándolo en la etapa final del experimento, siendo el T3 el más afectado desde etapas iniciales del experimentó.

La Figura 7B muestra la tasa de crecimiento del peso somático, siendo el T1 quien registró una mayor tasa $(\mathrm{T} 1=0.023 ; \mathrm{T} 2=0.016 ; \mathrm{T} 3=0.012 \mathrm{~g} /$ día $), \quad$ sin embargo no se encontró diferencia significativa entre T1 y T2, en contraste se encontró que el T3 que fue significativamente diferente al $\mathrm{T} 1$ y $\mathrm{T} 2$ desde la primera quincena. (Ver Anexo 2, Tabla 2). La tasa de crecimiento del soma fue variable en cada tratamiento, mostrando el T1 y T2 un comportamiento similar, a diferencia del T3 que presentó menores tasas de crecimiento. (Figura 7B).

La figura 8A muestra el crecimiento en peso seco de la gónada observándose un crecimiento oscilante, siendo el T1 el que presentó un mayor crecimiento
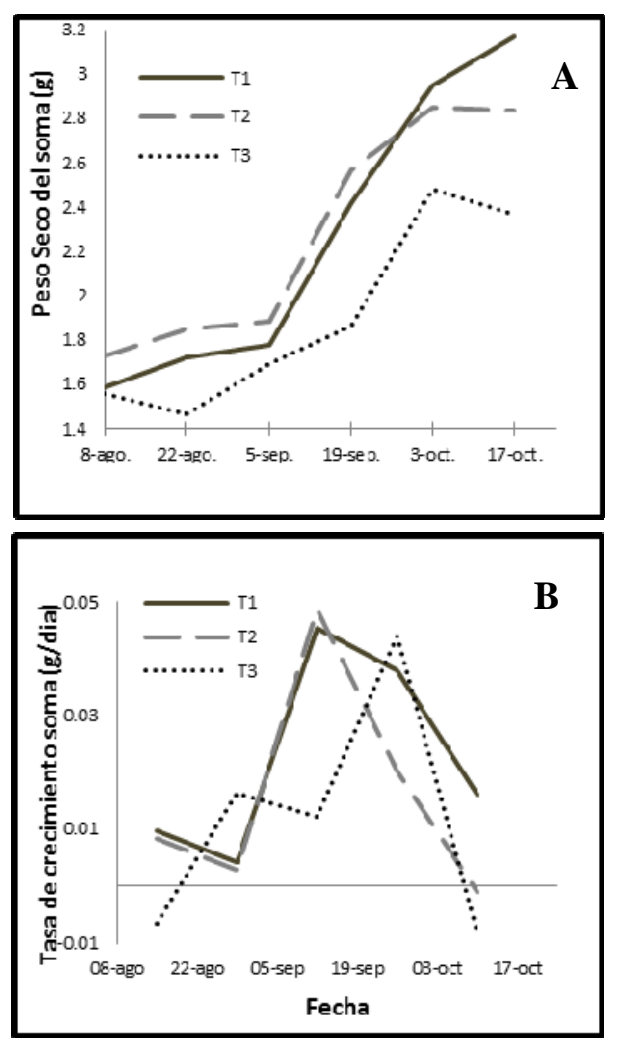

Figura 7. Peso seco medio del soma (g) (A) y Tasa de crecimiento del soma (g/día) (B) de A. purpuratus, playa Atenas, bahía de Paracas.

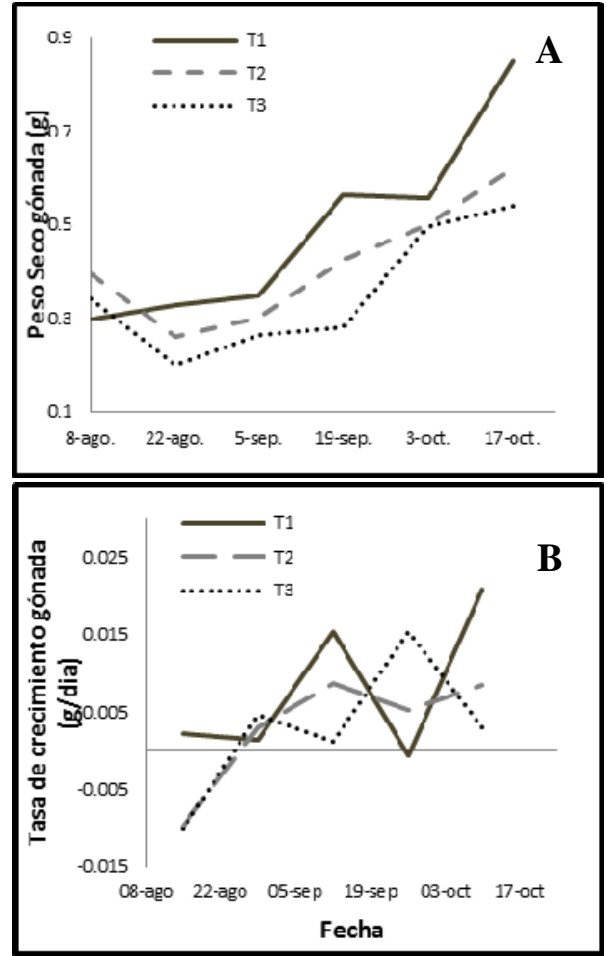

Figura 8. Peso seco medio de la gónada (g) (A) y Tasa de crecimiento de la gónada (g/día) (B), de A. purpuratus, playa Atenas, bahía de Paracas.

$(\mathrm{T} 1=0.0078 ; \quad \mathrm{T} 2=0.0031 ; \quad \mathrm{T} 3=0.0028 \quad \mathrm{~mm}), \quad$ sin embargo todos los tratamientos mostraron diferencias significativas, mostrando tasas de crecimiento muy variables en cada periodo de evaluación (Figura $8 \mathrm{~B}$ ). (Ver Anexo 4, Tabla 5).

Relación del índice gonado-somatico (IGS).

Los IGS mostraron crecimiento oscilante, pero con una tendencia a creciente, siendo el T2 y T3 los que mostraron diferencias significativas y presentaron

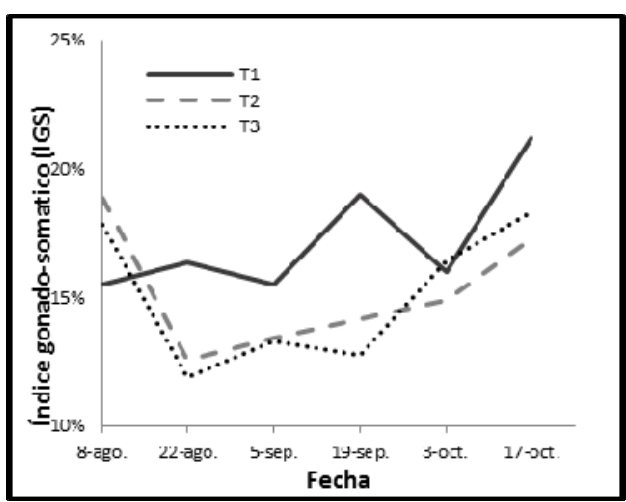

Figura 9. Índice gonado-somático de $A$. purpuratus registrado en Playa Atenas, bahía de Paracas. 
menores IGS desde el inicio y una recuperación lenta, con respecto al T1. (Figura 9). (Ver Anexo 4, Tabla 6).

Estimación de la supervivencia.

Se encontró una menor supervivencia en el tratamiento con mayor densidad de Ulva spp., T3, seguido del T2, lo que indica que Ulva spp., juega un rol importante afectando a la supervivencia de $A$. purpuratus. Así mismo se aprecia una mayor tasa de mortandad en las etapas finales de la evaluación. Las diferencias significativas entre tratamientos, solo se encontraron en el primer muestreo. (Fig. 10). (Ver Anexo 4, Tabla 7).

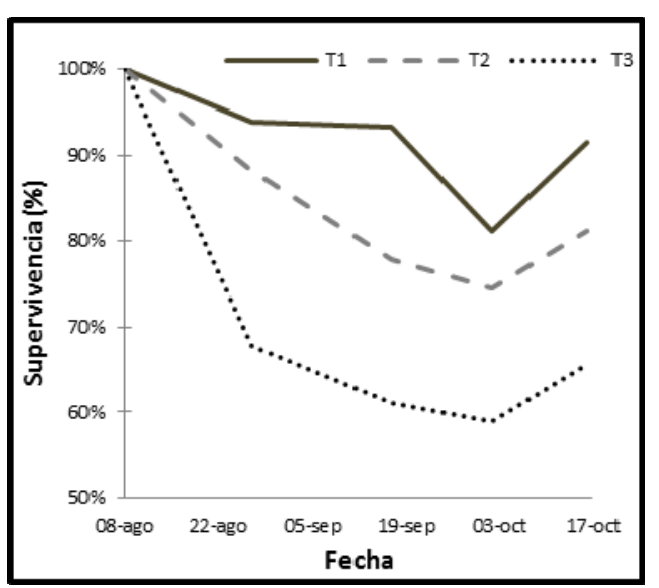

Figura 10. Supervivencia de A. purpuratus, playa Atenas, bahía de Paracas.

\section{Discusión.}

Si bien la velocidad de corrientes no pudo ser medida en cada tratamiento, es de suponer que la presencia de algas disminuya la velocidad de las corrientes y de esta manera afecte al crecimiento y/o supervivencia de A. purpuratus. En un estudio de Sara \& Mazzola, (2004) en dos zonas del mediterráneo (golfo de Castellammare y golfo de Gaeta), llegaron a la hipótesis que una baja velocidad de corriente $(<5 \mathrm{~cm} / \mathrm{s})$ en el golfo de Gaeta, era el factor limitante para poder tener una gran biomasa de Mytilus galloprovincialis, a pesar de presentar una alta disponibilidad de alimento y alta concentración de clorofila a. Ello concuerda con Aguirre (2004) y un estudio realizado frente a Parachique por Cabrera \& Mendo (2011), quienes encontraron que la corriente es uno de los factores limitantes para el desarrollo del soma y gónada de $A$. purpuratus, debido a que incrementa el flujo horizontal del sestón orgánico y de clorofila $a$.

Según los registros de la concentración de clorofila $a$ fueron mayores en los tratamientos con presencia de Ulva spp., T2 y T3, tanto en las mediciones realizadas al mediodía como en las registradas de forma continua, dichos tratamientos, presentaron menores tasas de crecimiento y supervivencia, demostrándonos que la alta disponibilidad de alimento no necesariamente se refleja en un mayor crecimiento y/o supervivencia, siendo posible que las corrientes jueguen un papel más importante, generando un mejor flujo del alimento, renovándolo y mejorando su calidad, como lo demuestra Aguirre (2004) y Cabrera \& Mendo, (2011).

Los tratamientos con presencia de Ulva spp., registraron valores más altos de temperatura sin embargo no se presentó gran diferencia entre los tratamientos, los valores se encontraron en el rango de tolerancia $\left(12-27^{\circ} \mathrm{C}\right)$, descritos por Mina et al., (2002) e IFOP (1993), para el desarrollo de A. purpuratus. Esta pequeña diferencia observada entre tratamientos pudo deberse a que Ulva spp., afectó el flujo de agua, disminuyendo su renovación y por lo tanto incrementando la temperatura al retener calor. El incremento de la variación de temperatura en los periodos finales $(\Delta$ T3-T1) podría deberse a la descomposición de la Ulva spp. (Ver anexo 1 y Figura 3C).

La concentración de oxígeno fue mayor en el T3, inclusive durante la noche, donde no existe producción de oxígeno por parte de Ulva spp., y otros microorganismos, lo que lleva a pensar que el consumo del oxígeno durante la noche es insignificante en relación a la producción de oxígeno por parte de Ulva spp., durante el día. A su vez dicho tratamiento presentó un mayor descenso de la concentración de oxígeno, lo cual puede atribuirse a la descomposición de Ulva spp., y al consumo de oxígeno por parte de la misma, propio de la respiración de la fase oscura tal como observó Burris (1977), en macroalgas y microalgas. Este descenso abrupto de la concentración de oxígeno en la noche se vio intensificado en la última quincena del experimento pudiendo ser la causante de la alta mortalidad encontrada en esa etapa.

Así mismo se encontraron valores por debajo de la concentración crítica $1.4 \mathrm{ml} / \mathrm{L}$ descrita por Yamashiro et al., (1990). Siendo la concentración del T1 inferior al T3. Sin embargo el T1 mostró una mayor tasa de crecimiento y supervivencia.

El crecimiento en altura fue significativamente mayor en el testigo (T1) que en T2 y T3, esto podría deberse a que inicialmente Ulva spp., limita el volumen de filtrado, al cubrir a las conchas con sus frondas, y aun siendo mayor la concentración de clorofila $a$ y oxígeno, esta no podía ser aprovechada por las conchas. Así mismo Ulva spp., tuvo un mayor efecto en las etapas finales debido a su asentamiento y acelerada descomposición, lo que pudo alterar la calidad del alimento, causando una disminución de la tasa de crecimiento, debido al rechazo del alimento, además la descomposición genera fango, el cual podría interferir con el filtrado de la concha, lo que implica un mayor costo de energía por el filtrado y un 
menor aprovechamiento del alimento. Investigadores como Griffiths \& Griffiths (1987) mencionan que el crecimiento en bivalvos también está regulado por la cantidad y calidad de alimento y según Broom \& Mason (1978) afirma que aunque exista un exceso de alimento este no necesariamente resultará en un incremento en el crecimiento.

El crecimiento en peso de la gónada, presentó un comportamiento irregular, siendo mayor esta variación en los tratamientos con presencia de Ulva spp. Esta variación en peso está relacionada con el ciclo reproductivo de $A$. purpuratus, que se puede observar a través del Índice Gonadosomatico (IGS). En este sentido Christiansen et al. (1974), menciona que los bivalvos filtradores ocasionalmente no evacuan todos los gametos sino solo parte de ellos, existiendo así periodos parciales o totales de liberación. En este estudio se observó un descenso inicial del peso de la gónada, en los tratamientos con presencia de Ulva spp., probablemente en respuesta ante el cambio de su medio, siendo el T3 el que presentó mayor variación en el peso de la gónada durante todo el periodo del experimento, lo que resultó en una baja tasa de crecimiento del soma y altura posiblemente debido al alto costo de energía destinada al crecimiento gonadal (Ver Anexo 2, Tabla 3 y Anexo 3, Tabla 4). Así mismo mencionan Griffiths \& Griffiths (1987) y Bricelj \& Shumway (1991) que el crecimiento somático disminuye o se paraliza cuando la energía es destinada a la gónada.

Si bien se encontró gran diferencia en el número de supervivientes entre los tratamientos, sólo fueron significativos en el primer y segundo muestreo, siendo el T3 el que sufrió una mayor mortandad en todos los muestreos, seguido del T2. Esto podría indicar que inicialmente la supervivencia de A. purpuratus se vio afectada por presencia de Ulva spp., posiblemente por el encierro que causaban sus frondas sin embargo las más altas mortandades se dieron en el tercer y cuarto muestreo (Ver Anexo 5 Figura 12) pero a pesar de ello, no mostraron diferencia significativa entre los tratamientos, esta baja supervivencia podría deberse a la fuerte irregularidad de la concentración de oxígeno, la cual disminuye bruscamente en la concentración en el T1 o testigo y mostró una alta variación en la concentración de día-noche del T3.

No se puede dejar de mencionar que en los últimos años se han realizado muchos estudios a nivel mundial, sobre el efecto de Ulva spp., debido a que han reemplazado a muchas algas y corales dominantes del ecosistema, como mencionan Valiela et al. (1997); Jousson et al. (2000); Meinesz et al. (2001). Dicha invasión genera cambios importantes en los ecosistemas, reduciendo la biodiversidad y alterando los parámetros ambientales. Entre ellos conocemos las defensas químicas por parte de Ulva spp., las cuales están compuestas por DMSP (dimetilsulfoniopropionato), cuyos metabolitos secundarios impiden la alimentación de diferentes herbívoros marinos, como describe Amy et al. (2006), generando efectos alelopáticos y tóxicos para las fases larvarias y adultas de muchos invertebrados y vertebrados marinos, (Guerriero et al., 1993; Paul et al., 2001; Jung y Pohnert, 2001; Paul \& Fenical, 1986; Lemee et al., 1993; Pedrotti et al., 1996; Nelson et al., 2003). Ello podría también explicar la reducción de los bancos de semilla de A. purpuratus en la bahía, como mencionan muchos maricultores.

Así mismo la gran proliferación de Ulva spp., genera una competencia intraespecífica llevando a una gran mortandad y su descomposición. En un estudio realizado por Chao Wang et al. (2012), encontraron que la descomposición de Ulva (Enteromorpha) libera cantidades considerables de nutrientes inorgánicos tóxicos, en particular el amonio que podrían causar mortandades masivas. Al respecto un estudio realizado por Widman et al. (2007), sobre la toxicidad ante la exposición de amoniaco no ionizado por 72 horas a A. irradians irradians $(7.2$ a $26.4 \mathrm{~mm})$, resultó en un $\mathrm{LC}_{50}$ de 52 ppm y mortalidad del $100 \%$ en concentraciones superiores a $122 \mathrm{ppm}$. Ello demuestra la toxicidad del amonio liberado por la descomposición de Ulva spp.

En este estudio no se tomaron muestras de agua, por lo que no se tiene datos sobre concentración de amonio o metabolitos secundarios de DMSP, lo que lleva a pensar que $A$. purpuratus sometido a la presencia de Ulva spp., (T2 y T3) inicialmente se vio afectado por sus defensas químicas (DMSP), el cual pudo provocar inicialmente una reducción del filtrado y por ende una disminución de la tasa de crecimiento.

Posteriormente la fuerte competencia intraespecifica de Ulva spp., provocó una mortandad de la misma y su descomposición, lo que dio lugar a la liberación de grandes cantidades de amonio, el cual podría ser altamente tóxico para el $A$. purpuratus como lo es para el A. irradians irradians, lo que llevó a incrementar las mortalidades en los tratamientos en presencia de Ulva spp., T2 y T3. Por ello en estudios futuros debería incluirse la medición de amonios para explicar con mayor profundidad su efecto sobre la concha de abanico sometida a altas densidades de Ulva spp.

\section{Conclusiones.}

- Cuando Ulva spp., se presenta en una densidad de $20 \mathrm{~kg} / \mathrm{m}^{2}$, afecta a la tasa de crecimiento y supervivencia de $A$. purpuratus.

- Los tratamientos T1 (sin presencia de Ulva spp.) y T2 (10kg Ulva spp. $\left./ \mathrm{m}^{2}\right)$, presentaron un mayor crecimiento que el T3 (20 kg Ulva spp. $\left./ \mathrm{m}^{2}\right)$ tanto en altura valvar, como en la gónada y soma.

- Los tratamientos T2 y T3 mostraron menores valores de Índice gónado-somático (IGS) desde el inicio de este estudio y una recuperación lenta siendo el T1 el que presentó un crecimiento continuo. 
- La supervivencia A. purpuratus se vio más afectada en el T3 $\left(20 \mathrm{~kg}\right.$ Ulva spp. $\left./ \mathrm{m}^{2}\right)$.

\section{Agradecimientos.}

La realización del presente trabajo de investigación fue gracias empresa PRISCO SAC, por prestarme parte de su concesión y las conchas para este estudio. Y a sus trabajadores por brindaron su apoyo durante los muestreos.

Agradezco al Dr. Mendo por el préstamo de los equipos y la confianza depositada en mí.

\section{Literatura citada.}

Aguirre H.E. 2004. Phytoplankton absorption, photosynthetic parameters, and primary production off Baja California, 1998. Deep-Sea Research II. 51: 799 816.

Bodelón O. G., Bernués M., Baltanás A. \& Montes C. (1994) Conductividad y Salinidad en los ecosistemas acuáticos del Parque Nacional de Doñana (SO España). Limnetica. 10: 27-31. Call Number: 626.

Bricelj V. \& Shumway M. 1991. Physiology: Energy acquisition and utilisation. Scallops Biology and Aquaculture. 7: 417-492.

Broom M.J. \& Mason J. 1978. Growth and spawning in the pectinidchlamy sopercularis in relation to temperature and phytoplankton concentration. Marine Biology. 47: 277-285.

Burris J.E. 1977. Photosynthesis, photorespiration, and dark respiration in eight species of algae. Marine Biology. 39: 371-379.

Cabrera P. \& Mendo J. 2011. Variación de la condición somática y reproductiva de la concha de abanico (Argopecten purpuratus), y su relación con las variables ambientales en la Bahía de Sechura. En Bases científicas y tecnológicas para incrementar la productividad del cultivo de concha de abanico en áreas de repoblamiento en la Bahía de Sechura. Libro de resúmenes. Universidad Nacional Agraria La Molina 26-31pp.

Cappelletti C. 1992. Estadística experimental. Editorial Agrovet, Buenos Aires. 513 p.

Chao W., Ren-Cheng Y. \& Ming-Jiang Z. 2012. Effects of the decomposing green macroalga Ulva (Enteromorpha) prolifera on the growth of four red-tide species harmful algae. Elsevier.16: 12-19.

Christiansen H.E., Cabrera E. \& Brodsky S.R. 1974 Ecología de las poblaciones de vieiras (Chlamys tehuelcha) en el golfo San Matías (Rio Negro, Argentina). I. Estudio histológico del ciclo reproductivo. Boletín del Instituto de Biología Marina. 225: 1-17.

Griffiths C.L. \& Griffiths R.J. 1987. Bivalvia. In Animal Energetics, Bivalvia through Reptilia, Pandian, T.J. and Vernberg, F.J., Eds. New York: Academic Press. 2:1-88.

Guerriero A., Marchetti F., D'ambrosio M., Senesi S., Dini F. \& Pietra F. 1993. New ecotoxicologically and biogenetically relevant terpenes of the tropical green seaweed Caulerpa taxifolia which is invading the Mediterranean. Helvetica Chimica Acta. 76: 855-864.

Hayden H.S. \& Waaland J.R. 2004. A molecular systematic study of WIY/ (Ulvaceae. Ulvales) from the northeast Pacific. Phycologia. 43: 364-382.
Hein M., Pedersen M.F. \& Sand-Jensen K. 1995. Sizedependent nitrogen uptake in micro- and macroalgae. Marine Ecology Progress Series. 118: 247-253.

Instrumento Financiero de Orientación de la Pesca. 1993. Investigación repoblamiento de recursos bentónicos área piloto IV región. Etapa V. Instituto de Fomento pesquero. sgi-ifop 93/8, Página 156.

Fong P., Donohoe R.M. \& Zedler J.B. 1994. Nutrient concentration in the tissue of the macroalgae Enteromorpha spp. as an indicator of nutrient history: an experimental evaluation using field microcosms. Marine Ecology Progress Series. 106: 273-281.

Jousson O., Pawlowski J., Zaninetti L., Zechman F.W., Din F., Guiseppe G., Woodfield R., Millar A. \& Meinesz A. 2000. Invasive alga reaches california - the alga has been identified that threatens to smother californian coastal ecosystems. Nature. 408: 157-158.

Jung V. \& Pohnert G. 2001. Rapid wound-activated transformation of the green algal defensive metabolite Caulerpenyne. Tetrahedron. 57: 7169-7172.

Lemee R., Pesando D., Durand-Clement M., Dubreuil A., Meinesz A., Guerriero A. \& Pietra F. 1993. Preliminary survey of toxicity of the green alga Caulerpa taxifolia introduced into the mediterranean. Journal of Applied Psicology. 5: 485-493.

Meinesz A., Belsher T., Thibaut T., Antolic B., Ben Mustapha K., Boudouresque C.F., Chiaverini D., Cinelli F., Cottalorda J.M., Djellouli A., El Abed A., Orestano C., Grau A.M., Ivesa L., Jaklin A., Langar H., MassutiPascual E., Peirano A., Tunesi L., De Vaugelas J., Zavodnik N. \& Zuljevic A. 2001. The introduced alga Caulerpa taxifolia continuesto spread in the Mediterranean. Biological Invasions. 3: 201-210.

Naldi M. 2002. Nitrate uptake and storage in the seaweed Ulva rigida C. agardh in relation to nitrate availability and thallus nitrate content in a eutrophic coastal lagoon (sacca di goro, po river Delta, Italy). Journal of Experimental Marine Biology and Ecology. 269: 65- 83.

Mina L., Bandin R. \& Zavala J. 2002. Monitoreo de algunas variables ambientales y abundancia larval de Argopecten purpuratus en la Reserva Nacional de Paracas entre enero del 2000 y febrero del 2001. Páginas. 40 - 44. En: J. Mendo y M. Wolf (editores.), "Bases ecológicas y socioeconómicas para el manejo de los recursos vivos de la reserva nacional de Paracas" Lima - Perú.

Nelson T.A., Lee D.J. \& Smith B.C. 2003. Are"green tides" harmful algal blooms? Toxic properties of water-soluble extracts from two bloom-forming macroalgae, Ulva fenestrata and Ulva riaobscura (Ulvophyceae). Journal of Phycology. 39: 874-879.

Paul V.J. \& Fenical W. 1986. Chemical defense in tropical green algae, order caulerpales. Marine Ecology Progress Series. 34: 157-169.

Paul V.J., Cruz R.E. \& Thacker R.W. 2001. Chemical mediation of macroalgal-herbivore interactions: ecological and evolutionary perspectives. En: McClintock, J.B. and Baker, B.J., (eds.). Marine Chemical Ecology. CRC. 227-265.

Peckol P., Demeo-Anderson B. \& Rivers J. 1994. Growth, nutrient uptake capacities, and tissue constituents of the macroalgae Gracilaria tikvahiae and Cladophora vagabunda, related to site-specific nitrogen loading rates. Marine Biology. 121: 175-185. 
Pedersen M.F. \& Borum J. 1997. Nutrient control of estuarine algae: growth strategy and the balance between nitrogen requirements and uptake. Marine Ecology Progress Series. 161: 155-163.

Pedrotti M.L., Marchi B. \& Lemee R. 1996. Effects of Caulerpa taxifolia secondary metabolites on the embryogenesis, larval development and metamorphosis of the sea urchin Paracentrotus lividus. Oceanologica Acta. 19: 255-262.

Sara G. \& Mazzola A. 2004. The carrying capacity for Mediterranean bivalve suspension feeders: Evidence from analysis of food availability and hydrodynamics and their integration into a local model. Ecological Modelling. 179: 281-296.

Valiela I., Mcclennand J., Hauxwell J., Behr P.J., Hersh D. \& Foreman K. 1997. Macroalgal blooms in shallow estuaries: Controls and ecophysiological and ecosystem consequences. Limnology and Oceanography. 42:11051118.

Valiela I., Mcclennand J., Hauxwell J., Behr P.J., Hersh D. \& Foreman K. 1997b. Macroalgal blooms in shallow estuaries: Controls and ecophysiological and ecosystem consequences. Limnology and Oceanography. 42: 110518.

Widman Jr. J.C., Meseck S.L., Sennefelder G. \& Veilleux D.J. 2007. Toxicity of un-ionized ammonia, nitrite, and nitrate to juvenile bay scallops, A. irradians irradians. Archives of Environmental Contamination \& Toxicology. 54: 460.

Yamashiro C., Rubio J., Jurado E., Auza E., Maldonado M., Ayon P. \& Antonietti E. 1990. Evaluación de la población de concha de abanico (A. purpuratus) en la bahía Independencia, Pisco, Perú. Informeins. MarperúCallao. (98): 58.

\section{ANEXOS}

ANEXO 1: Variación de temperatura T3-T1 $\left({ }^{\circ} \mathrm{C}\right)$.

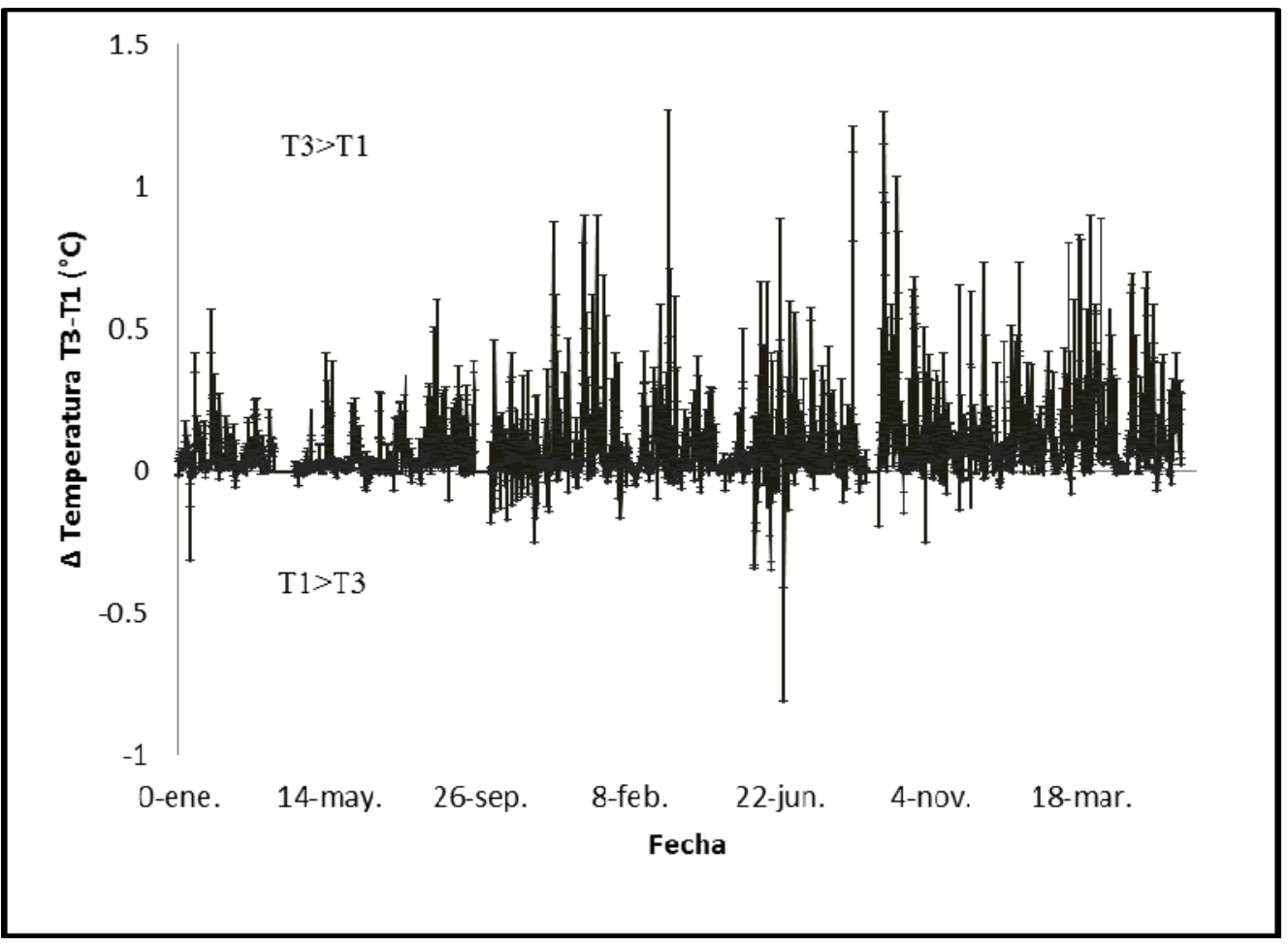

Figura 11. Variación de temperatura T3-T1 $\left({ }^{\circ} \mathrm{C}\right)$.

*Los valores positivos de la gráfica indican superioridad de temperatura del T3 con respecto al T1.

*Los registros fueron tomados cada 30 minutos para cada tratamiento, existiendo mayores variaciones en el periodo final. 
ANEXO 2: Prueba de comparación de medias (Tukey).

Tabla 1. Prueba de comparación de medias (Tukey) para la variable altura de A. purpuratus cultivadas en corrales en dos diferentes densidades de Ulva spp., y sin Ulva spp., en la zona de Paracas.

\begin{tabular}{lll}
\hline Muestreo & P - valor & Significancia \\
\hline 5-Sept-11 & & \\
T1-T2 & .790 & No \\
T1-T3 & .072 & No \\
T2-T3 & .014 & Si \\
19-Sept-11 & & \\
T1-T2 & .553 & No \\
T1-T3 & .000 & Si \\
T2-T3 & .001 & Si \\
3-Oct-11 & & \\
T1-T2 & .036 & $S i$ \\
T1-T3 & .000 & $S i$ \\
T2-T3 & .000 & $S i$ \\
17-Oct-11 & & \\
T1-T2 & .000 & Si \\
T1-T3 & .000 & Si \\
T2-T3 & .000 & Si \\
\hline T1 =Control, T2 $=10 \mathrm{~kg} \mathrm{y} \mathrm{T3}=20 \mathrm{~kg} \mathrm{de} \mathrm{Ulva} \mathrm{spp} . / \mathrm{m}^{2}$.
\end{tabular}

Tabla 2. Prueba de comparación de medias (Tukey) para la variable peso seco somático de A. purpuratus cultivadas en corrales en dos diferentes densidades de Ulva spp., y sin Ulva spp., en la zona de Paracas.

\begin{tabular}{lll}
\hline Muestreo & P - valor & Significancia \\
\hline 22-Ago-11 & & \\
T1-T2 & .429 & $\mathrm{No}$ \\
T1-T3 & .037 & $\mathrm{Si}$ \\
T2-T3 & .001 & $\mathrm{Si}$ \\
19-Sept-11 & & \\
T1-T2 & .629 & $\mathrm{No}$ \\
T1-T3 & .004 & $\mathrm{Si}$ \\
T2-T3 & $.000)$ & $\mathrm{Si}$ \\
3-Oct-11 & & \\
T1-T2 & .776 & $\mathrm{No}$ \\
T1-T3 & .003 & $\mathrm{Si}$ \\
T2-T3 & .021 & $\mathrm{Si}$ \\
17-Oct-11 & & \\
T1-T2 & .095 & $\mathrm{No}$ \\
T1-T3 & .000 & $\mathrm{Si}$ \\
T2-T3 & .01 & $\mathrm{Si}$ \\
\hline T1 $=$ Control, T2 $=10 \mathrm{~kg}$ y T3 $=20 \mathrm{~kg}$ de Ulva $\mathrm{spp} . / \mathrm{m}^{2}$.
\end{tabular}

Tabla 3. Desviación estándar de $A$. purpuratus cultivadas en corrales en dos diferentes densidades de Ulva spp., y sin Ulva spp., en la zona de Paracas. Altura valvar (mm) (A), Peso seco somático (g) (B), Peso seco de gónada (g) (C).

\begin{tabular}{cccc}
\hline \multicolumn{4}{c}{ A. Altura valvar $(\mathrm{mm})$} \\
\hline Fecha & T1 & T2 & T3 \\
22-ago & 0.25 & 0.3 & 0.22 \\
05-sep & 0.34 & 0.32 & 0.3 \\
19-sep & 0.29 & 0.34 & 0.21 \\
03-oct & 0.22 & 0.28 & 0.24 \\
17-oct & 0.18 & 0.27 & 0.22 \\
\hline \multicolumn{5}{c}{ B. Peso seco somático $(\mathrm{g})$} \\
\hline Fecha & T1 & T2 \\
08-ago & 0.36 & 0.5 & T3 \\
22-ago & 0.41 & 0.47 & 0.3 \\
05-sep & 0.44 & 0.4 & 0.26 \\
19-sep & 0.42 & 0.98 & 0.39 \\
03-oct & 0.53 & 0.45 & 0.61 \\
17-oct & 0.7 & 0.68 & 0.43 \\
\hline \multicolumn{5}{c}{ C. Peso seco Gónadal (g) } \\
\hline Fecha & T1 & T2 \\
08-ago & 0.11 & 0.13 & T3 \\
22-ago & 0.08 & 0.12 & 0.11 \\
05-sep & 0.18 & 0.19 & 0.1 \\
19-sep & 0.21 & 0.22 & 0.13 \\
03-oct & 0.17 & 0.15 & 0.24 \\
17-oct & 0.22 & 0.31 & 0.21 \\
\hline T1 =Control, T2 $=10 \mathrm{~kg}$ y T3 $=20 \mathrm{~kg} \mathrm{de}$ Ulva spp. $/ \mathrm{m}^{2}$.
\end{tabular}

$\mathrm{T} 1=$ Control, $\mathrm{T} 2=10 \mathrm{~kg}$ y T3 $=20 \mathrm{~kg}$ de Ulva spp. $/ \mathrm{m}^{2}$. 
ANEXO 3: Desviación estándar de la tasa de crecimiento.

Tabla 4. Desviación estándar de la tasa de crecimiento de A. purpuratus cultivadas en corrales en dos diferentes densidades de Ulva spp., y sin Ulva spp., en la zona de Paracas, entre el 08 de agosto y 17 de octubre del 2011.

\begin{tabular}{|c|c|c|c|c|}
\hline \multicolumn{5}{|c|}{ A. Del 08 al 22 de agosto del 2011} \\
\hline & $\begin{array}{l}\text { Talla } \\
(\mathbf{m m})\end{array}$ & $\begin{array}{c}\text { Peso } \\
\text { somático (g) }\end{array}$ & $\begin{array}{c}\text { Peso } \\
\text { sexual (g) }\end{array}$ & $\begin{array}{c}\text { Peso } \\
\text { total (g) }\end{array}$ \\
\hline T1 & 0.379 & 0.674 & 0.160 & 0.721 \\
\hline $\mathbf{T 2}$ & 0.426 & 0.430 & 0.117 & 0.489 \\
\hline T3 & 0.315 & 0.358 & 0.143 & 0.421 \\
\hline \multicolumn{5}{|c|}{ B. Del 22 de agosto al 05 de septiembre del 2011} \\
\hline & $\begin{array}{l}\text { Talla } \\
(\mathbf{m m})\end{array}$ & $\begin{array}{c}\text { Peso } \\
\text { somático (g) }\end{array}$ & $\begin{array}{c}\text { Peso } \\
\text { sexual (g) }\end{array}$ & $\begin{array}{c}\text { Peso } \\
\text { total (g) }\end{array}$ \\
\hline T1 & 0.395 & 0.687 & 0.244 & 0.740 \\
\hline $\mathbf{T} 2$ & 0.518 & 0.552 & 0.194 & 0.683 \\
\hline T3 & 0.329 & 0.352 & 0.137 & 0.419 \\
\hline \multicolumn{5}{|c|}{ C. Del 05 al 19 de septiembre del 2011} \\
\hline & $\begin{array}{l}\text { Talla } \\
\text { (mm) }\end{array}$ & $\begin{array}{c}\text { Peso } \\
\text { somático (g) }\end{array}$ & $\begin{array}{c}\text { Peso } \\
\text { sexual (g) }\end{array}$ & $\begin{array}{c}\text { Peso } \\
\text { total (g) }\end{array}$ \\
\hline T1 & 0.553 & 1.084 & 0.290 & 1.250 \\
\hline T2 & 0.488 & 0.696 & 0.311 & 0.934 \\
\hline T3 & 0.311 & 0.498 & 0.152 & 0.587 \\
\hline \multicolumn{5}{|c|}{ D. Del 19 de septiembre al 03 de octubre del 2011} \\
\hline & $\begin{array}{l}\text { Talla } \\
\text { (cm) }\end{array}$ & $\begin{array}{c}\text { Peso } \\
\text { somático (g) }\end{array}$ & $\begin{array}{c}\text { Peso } \\
\text { sexual (g) }\end{array}$ & $\begin{array}{c}\text { Peso } \\
\text { total (g) }\end{array}$ \\
\hline T1 & 0.498 & 1.142 & 0.304 & 1.345 \\
\hline $\mathbf{T} 2$ & 0.403 & 0.731 & 0.248 & 0.790 \\
\hline T3 & 0.319 & 0.717 & 0.256 & 0.809 \\
\hline \multicolumn{5}{|c|}{ E. Del 11 al 17 de octubre del 2011} \\
\hline & $\begin{array}{l}\text { Talla } \\
(\mathbf{m m})\end{array}$ & $\begin{array}{c}\text { Peso } \\
\text { somático (g) }\end{array}$ & $\begin{array}{c}\text { Peso } \\
\text { sexual (g) }\end{array}$ & $\begin{array}{c}\text { Peso } \\
\text { total (g) }\end{array}$ \\
\hline T1 & 0.385 & 0.773 & 0.335 & 1.030 \\
\hline T2 & 0.265 & 0.894 & 0.279 & 0.999 \\
\hline T3 & 0.293 & 0.824 & 0.318 & 1.002 \\
\hline
\end{tabular}

ANEXO 4: Comparación de medias (Tukey).

Tabla 5. Resultados de las prueba de comparación de medias (Tukey) para la variable peso seco de la gónada de $A$. purpuratus cultivadas en corrales en dos diferentes densidades de Ulva spp., y sin Ulva spp., en la zona de Paracas.

\begin{tabular}{lll}
\hline Muestreo & P - valor & Significancia \\
\hline 22-Ago-11 & & \\
T1-T2 & .039 & $S i$ \\
T1-T3 & .000 & $S i$ \\
T2-T3 & .082 & No \\
19-Set-11 & & \\
T1-T2 & .012 & $S i$ \\
T1-T3 & .000 & $S i$ \\
T2-T3 & .009 & $S i$ \\
17-Oct-11 & & \\
T1-T2 & .002 & $S i$ \\
T1-T3 & .000 & Si \\
T2-T3 & .446 & No \\
\hline T1 $=$ Control, T2 $=10 \mathrm{~kg} \mathrm{y} \mathrm{T3}=20 \mathrm{~kg}$ de Ulva $\mathrm{spp} . / \mathrm{m}^{2}$.
\end{tabular}

Tabla 6. Resultados de las prueba de comparación de medias (Tukey) para la variable índice gonado-somatico de $A$. purpuratus cultivadas en corrales en dos diferentes densidades de Ulva spp y sin Ulva spp, en la zona de Paracas.

\begin{tabular}{lll}
\hline Muestreo & P-valor. & Significancia \\
\hline 22-Ago-11 & & \\
T1-T2 & .013 & SI \\
T1-T3 & .003 & SI \\
T2-T3 & .864 & NO \\
19-Sept-11 & & \\
T1-T2 & .002 & SI \\
T1-T3 & .000 & SI \\
T2-T3 & .556 & NO \\
17-Oct-11 & & \\
T1-T2 & .008 & SI \\
T1-T3 & .068 & NO \\
T2-T3 & .699 & NO \\
\hline T1 $=$ Control, T2 $=10 \mathrm{~kg} \mathrm{y} \mathrm{T3}=20 \mathrm{~kg}$ de Ulva spp $/ \mathrm{m}^{2}$.
\end{tabular}


Tabla 7. Resultados de las prueba de comparación de medías (Tukey) de la supervivencia de $A$. purpuratus cultivadas en corrales en dos diferentes densidades de Ulva spp., y sin Ulva spp., en la zona de Paracas.

\begin{tabular}{lll}
\hline Muestreo & P-valor. & Significancia \\
\hline 27 Ago 11 & & \\
T1-T2 & .457 & No \\
T1-T3 & .002 & $S i$ \\
T2-T3 & .008 & $S i$ \\
17 Sept 11 & & \\
T1-T2 & .345 & No \\
T1-T3 & .045 & Si \\
T2-T3 & .304 & No \\
2 Oct 11 & & \\
T1-T2 & .701 & No \\
T1-T3 & .073 & No \\
T2-T3 & .210 & No \\
16 Oct 11 & & \\
T1-T2 & .513 & No \\
T1-T3 & .063 & No \\
T2-T3 & .274 & No \\
\hline T1 =Control, T2 $=10 \mathrm{~kg} \mathrm{y} \mathrm{T3=20kg} \mathrm{de} \mathrm{Ulva} s p p / \mathrm{m}^{2}$.
\end{tabular}

\section{ANEXO 5. Tasa de mortalidad diaria.}

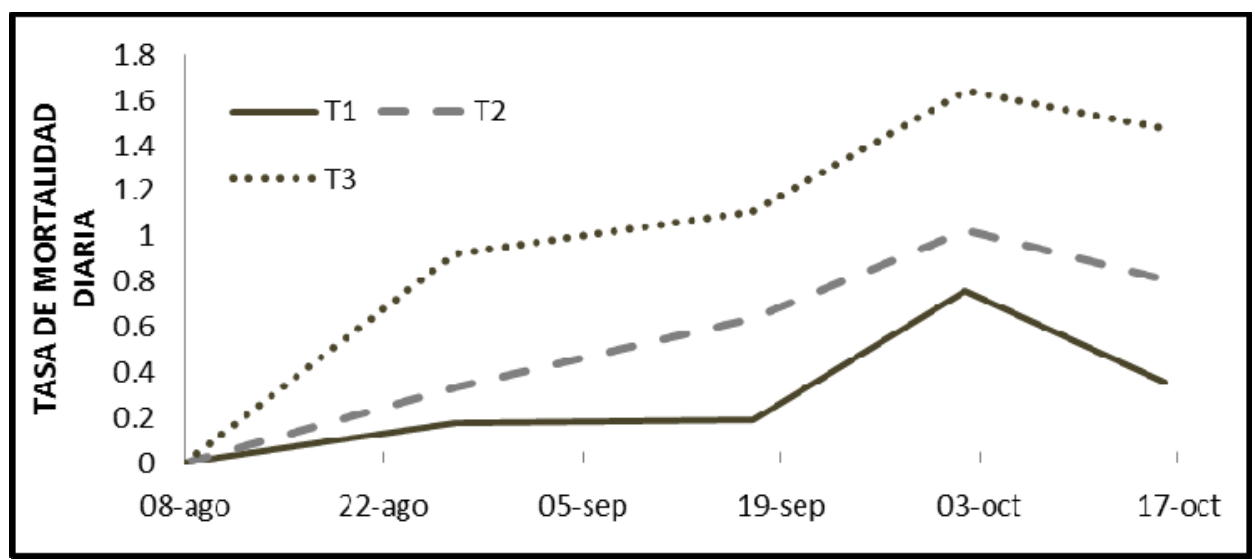

Figura 12. Tasa de mortalidad diría del T1, T2 y T3.

La figura 12 muestra, la muerte diría en cada tratamiento de cada periodo de muestreo. Se observó un incremento en la mortandad diaria en etapas finales.

${ }^{1}$ Av. Nueva Toledo 242 B, Cieneguilla oedanac@gmail.com

${ }^{2}$ Facultad de Pesquería, Universidad Nacional Agraria La Molina Av. La Molina s/n Lima - Perú. Correo electrónico:jmendo@lamolina.edu.pe 